\title{
SURFACTANT DYNAMICS FROM THE ARNOLD PERSPECTIVE
}

\author{
JONATHAN JENKINS ${ }^{1}$, CAROLYN LEE ${ }^{1}$, YUXUAN LIU ${ }^{1}$, ETHAN LU ${ }^{1}$, DESMOND REED ${ }^{2}$ \\ ${ }^{1}$ Department of Mathematical Sciences, Carnegie Mellon University, Pittsburgh, PA \\ ${ }^{2}$ Department of Physics, Carnegie Mellon University, Pittsburgh, PA
}

\begin{abstract}
In 1966, V. Arnold established an important connection between the incompressible Euler equations and a particular set of geodesic flows, using variational techniques to characterize the latter as solutions to the former. Motivated by his results, we investigate a series of similar PDEs characterizing constrained critical points of action functionals, paying particular interest to those associated with surfactant dynamics. Starting with the Arnold functional, we introduce various complications, adding terms associated to potential energies, surface tension, and surfactant momentum to derive different PDEs.
\end{abstract}

\section{INTRODUCTION/BACKGROUND}

One of the goals of this paper is to study a model of surfactants using the Euler system. Surfactants (surface-active agents, e.g. detergents, emulsifiers, and soap bubbles) adhere to the surface of fluids and have the ability to change magnitude of surface tension. Their behavior is of importance in the cosmetic industry, ore extraction and other areas (see for instance, [HLT08]). They also play a key role in biology, preventing the collapse of lungs during normal breathing (see [Hil99]). For a more in-depth explanation of surfactant physics and their key properties, see [EBW91] and the review [Sar96].

Now we'll discuss the setup. Let $\Omega$ be a bounded, open, connected subset of $\mathbb{R}^{n}$ with $\Sigma:=\partial \Omega$ smooth and set $\nu: \Sigma \rightarrow \mathbb{R}^{n}$ to be the associated outward pointing unit normal. See Figure 1 for a diagram of the unit normal. The incompressible Euler equations, first discovered by Euler in 1757, are an important set of PDEs characterizing the evolution of inviscid fluids. Assuming that such a fluid has constant, uniform density, and is trapped in a fixed container set $\Omega$, the equations read

$$
\begin{cases}\partial_{t} u+u \cdot \nabla u+\nabla p=0 & \text { on } \Omega \\ \operatorname{div} u=0 & \text { on } \Omega \\ u \cdot \nu=0 & \text { on } \Sigma\end{cases}
$$

where $u: \Omega \times[0,1] \rightarrow \mathbb{R}^{n}$ is the Eulerian velocity of the fluid and $p: \Omega \times[0,1] \rightarrow \mathbb{R}^{n}$ is the pressure exerted on the fluid.

(We consider only $t \in[0,1]$ for simplicity, since more general time domains can be obtained by rescaling.)

Let $\eta$ be the flow map associated to $u$ via

$$
\left\{\begin{array}{l}
\eta(x, 0)=x \\
\partial_{t} \eta(x, t)=u(\eta(x, t), t)
\end{array}\right.
$$

We note that the divergence free condition on $u$ in Equation (1.1) is equivalent to $\eta$ being volume preserving (see Theorem 2.3) and also that the last equation guarantees that the fluid does not "escape the boundary," so that the image of $\eta$ is always equal to $\Omega$.

E-mail address: $\{$ jtjenkin, cnl2, yuxuanli, ethanlu, dhreed, iantice\}@andrew.cmu.edu.

Key words and phrases. Surfactant dynamics, Arnold Interpretation, Geodesic flow.

Advisor Ian Tice was supported by an NSF CAREER Grant (DMS \#1653161). J. Jenkins, C. Lee, Y. Liu, E. Lu, D. Reed were supported by the summer research support provided by this grant. 
If in addition the fluid is moving freely (the boundary is not constant) and has a constant surface tension $\sigma \geqslant 0$ (where surface tension is the force of the surface adhering the bulk of the fluid), the equations become

$$
\begin{cases}\partial_{t} u+u \cdot \nabla u+\nabla p=0 & \text { on } \Omega(t) \\ \operatorname{div} u=0 & \text { on } \Omega(t) \\ p=-\sigma H & \text { on } \Sigma(t)\end{cases}
$$

where $\Omega(t)$ and $\Sigma(t)$ respectively describe the fluid and its boundary at time $t$ (see Figure 2) and $H=$ $-\operatorname{div} \nu$ is the mean curvature operator on $\Sigma(t)$ (average of curvature in all directions).

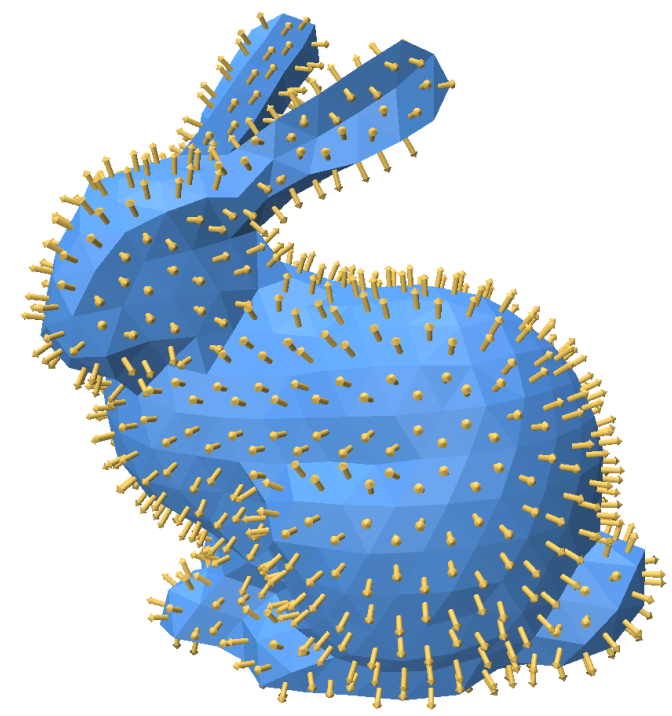

FIGURE 1. Outward pointing unit normal on $\Sigma$.

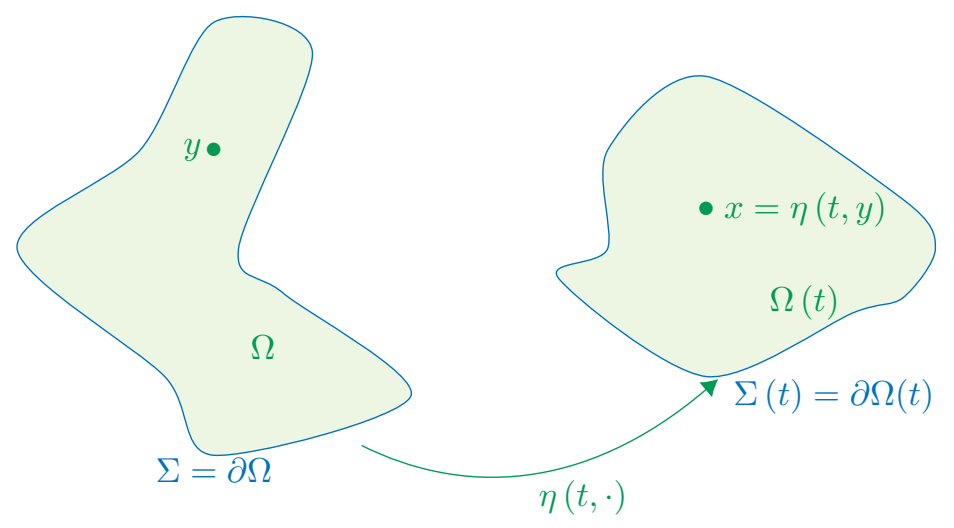

FiguRE 2. Flow map with free boundary

Historically, numerous connections were made between PDEs and energy functionals. One famous example is the Euler-Lagrange Equation, which we state below. (See Section 8.1.2 of [Eva10] for a derivation.) 
Theorem 1.1. (Euler-Lagrange Equation)

Let $\Omega \subseteq \mathbb{R}^{n}$ be bounded and open with a smooth boundary. Suppose $E: C_{b}^{\infty}(\Omega) \rightarrow \mathbb{R}$ is given by

$$
E(u):=\int_{\Omega} L(x, u(x), \nabla u(x)) d x
$$

where $L(x, z, p): \Omega \times \mathbb{R} \times \mathbb{R}^{n} \rightarrow \mathbb{R}$ is smooth. Assume further that there exists some $u \in C_{b}^{\infty}(\Omega)$ that minimizes this energy, i.e. $u=\arg \min _{C_{b}^{\infty}(\Omega)} E$. Then $u$ must satisfy

$$
\partial_{z} L(x, u(x), \nabla u(x))=\operatorname{div} \nabla_{p} L(x, u(x), \nabla u(x))
$$

The standard Euler-Lagrange equation is an unconstrained variational optimization problem, where energy minimizers are allowed to take on arbitrary values. However, especially in the context of fluid dynamics, the constrained versions of these optimization problems (where inputs to the energy are required to lie on some constraint manifold) are of more interest.

A particularly notable result in this context is due to Vladimir Arnold, who established the connection between the incompressible Euler equations and the Arnold energy functional (which will be introduced in Equation (1.6)) (see [Arn66, AK98]).

In this paper, motivated by these results, the work of Shatah and Zeng in [SZ07], and results like those surveyed in [KMM20], we make various modifications to the Arnold functional related to surfactants and study the resulting PDEs.

We begin first by defining the relevant function spaces that we'll be working in.

Definition 1.2. Given a fixed reference frame $\Omega$ as above, we define FDiff $(\Omega) \subseteq L^{2}\left(\Omega ; \mathbb{R}^{n}\right)$ to be the space of volume and orientation preserving smooth diffeomorphisms on $\Omega$; that is,

$$
F \operatorname{Diff}(\Omega):=\left\{\eta: \Omega \rightarrow \mathbb{R}^{n} \mid \eta \text { is a smooth volume/orientation preserving diffeomorphism }\right\}
$$

Given any such $\eta \in \operatorname{FDiff}(\Omega)$, one can naturally view it as encoding a possible evolution of the set $\Omega$, where $\eta$ provides Lagrangian coordinates.

In presenting Arnold's original work, we'll also need to work with a slightly smaller subset of FDiff $(\Omega)$ where the boundary is fixed. Although we won't be working extensively in this space, we present its definition here for completeness.

Definition 1.3. We define Diff $(\Omega)$ to be the space of smooth diffeomorphisms of $\Omega$ onto itself; that is,

$$
\operatorname{Diff}_{0}(\Omega):=\{\eta: \Omega \rightarrow \Omega \mid \eta \in F \operatorname{Diff}(\Omega)\}
$$

As remarked above, this space corresponds to the fixed boundary case, whereas the previous definition allows for free boundary.

Now in the remainder of this section, we state Arnold's previous results on geodesic flows in Diff $0(\Omega)$ and our main results about action minimizing flows on $\operatorname{FDiff}(\Omega)$.

Remark. Throughout the rest of this paper, we'll largely be taking for granted existence and smoothness of the different functions that we'll be working with, implicitly assuming everything is $C^{\infty}$ unless otherwise stated. While explicitly tracking the minimal degrees of regularity needed can be done (and is even present in many of the technical tools we present), these assumptions greatly facilitate the calculations being done without needing to appeal to e.g. the theory of distributions. In a similar vein, in our main results, we'll be assuming a priori that critical points of the action functionals under consideration exist, and will not discuss the necessary assumptions for their existence nor regularity.

Theorem 1.4. (Arnold, 1966)

Fix $\eta_{0}, \eta_{1} \in \operatorname{Diff}_{0}(\Omega)$, and let $\tilde{X}$ be the space of flows with initial configuration $\eta_{0}$ and terminal configuration $\eta_{1}$; that is,

$$
\tilde{X}:=\left\{\eta \in C^{1}\left([0,1] ; \operatorname{Diff}_{0}(\Omega)\right) \mid \eta(0)=\eta_{0}, \eta(1)=\eta_{1}\right\}
$$


Then minimizers (if they exist) of the energy functional $E: \tilde{X} \rightarrow \mathbb{R}^{+}$defined via

$$
E(\eta)=\int_{0}^{1} \int_{\Omega} \frac{1}{2}\left|\partial_{t} \eta\right|^{2} d x d t
$$

must satisfy the incompressible Euler equations with fixed boundary and uniform constant density

$$
\begin{cases}\partial_{t} u+u \cdot \nabla u+\nabla p=0 & \text { on } \Omega \\ \operatorname{div} u=0 & \text { on } \Omega \\ u \cdot \nu=0 & \text { on } \Sigma\end{cases}
$$

where $\Sigma=\partial \Omega, u: \Omega \times[0,1] \rightarrow \mathbb{R}^{n}$ is the Eulerian velocity defined via $u(\eta(x, t), t)=\partial_{t} \eta(x, t)$ and $p:[0,1] \rightarrow C^{\infty}(\Omega)$ is the pressure term.

Remark. We call such minimizers geodesic flows, as their associated optimality condition is analogous to the optimality condition for geodesics on manifolds in Euclidean space, which minimize

$$
E_{g}(\eta)=\int_{0}^{1} \frac{1}{2}\left|\partial_{t} \eta_{g}\right|^{2} d t
$$

where $\eta_{g}:[0,1] \rightarrow M$ is a path along some manifold $M$. The $g$ subscript indicates that for this remark, we are talking about geodesics instead of flow maps.

Here, the only modification being made is to the Riemannian metric:

$$
\left\|\partial_{t} \eta\right\|^{2}:=\int_{\Omega}\left|\partial_{t} \eta\right|^{2} d x
$$

Note that the energy functional is precisely integral of this term over time.

Now switching to the free boundary case, we introduce various additional modifications to Arnold's functional, which in turn lead to new PDEs. For simplicity, we define

$$
\Omega(t):=\eta(\Omega, t) \text { and } \Sigma(t):=\eta(\Sigma, t)=\eta(\partial \Sigma, t)
$$

so that the image of $\Omega$ at time $t$ is $\Omega(t)$ with boundary given by $\Sigma(t)$.

The first additional term we introduce is a globally defined potential energy term $\varphi$, which can compensate for other forces acting on the fluid such as gravity or an electromagnetic field. We also consider complications of the fluid itself, where we allow now for the fluid to have variable density. Defining $\bar{\rho}: \Omega \rightarrow \mathbb{R}^{+}$ to be the Lagrangian density of the fluid, we can then also set $\rho: \Omega(t) \times[0,1] \rightarrow \mathbb{R}^{+}$to be the Eulerian density of the fluid, noting that these two quantities are related by

$$
\rho(\eta(x, t), t)=\bar{\rho}(x)
$$

After considering all of these effects, we arrive at an action functional similar to one investigated by Shatah and Zeng in Section 2.1 of [SZ07], but that notably includes these two extra degrees of complexity.

Remark. Note that since we have introduced and subtracted a potential term, we are now considering a wider class of action functionals, which generalize the energy functionals/geodesic distances seen in Arnold's work. In particular, since this new energy is given by the difference of the kinetic and potential energies, we're now considering the Lagrangian of the system.

Theorem 1.5. Fix $\eta_{0}, \eta_{1} \in F \operatorname{Diff}(\Omega)$, and let $X$ be the space of flows with initial configuration $\eta_{0}$ and terminal configuration $\eta_{1}$; that is,

$$
X:=\left\{\eta \in C^{1}([0,1] ; F \operatorname{Diff}(\Omega)) \mid \eta(0)=\eta_{0}, \eta(1)=\eta_{1}\right\}
$$

Then given any $\varphi \in C^{1}\left(\mathbb{R}^{n}\right)$ and $\bar{\rho}: \Omega \rightarrow \mathbb{R}^{+}$, critical points (if they exist) of the action $E: X \rightarrow \mathbb{R}$ defined via

$$
E(\eta)=\int_{0}^{1} \int_{\Omega} \frac{\bar{\rho}}{2}\left|\partial_{t} \eta\right|^{2}-\varphi(\eta) d x d t
$$


must satisfy the incompressible Euler equations with free boundary and surface tension:

$$
\begin{cases}\rho\left(\partial_{t} u+u \cdot \nabla u\right)+\nabla p=-\nabla \varphi & \text { on } \Omega(t) \\ \operatorname{div} u=0 & \text { on } \Omega(t) \\ p=0 & \text { on } \Sigma(t)\end{cases}
$$

where $u: \Omega(t) \times[0,1] \rightarrow \mathbb{R}^{3}$ is the Eulerian velocity defined via $u(\eta(x, t), t)=\partial_{t} \eta(x, t)$ and $p:[0,1] \rightarrow$ $C^{\infty}(\Omega(t))$ is the pressure.

Focusing on the dynamics of the boundary, we can also introduce a boundary dependent term $\sigma$ that takes into account surface tension by integrating the surface tension against the surface. The surface tension essentially acts as a potential energy just as $\varphi$ does.

Theorem 1.6. Fix $\eta_{0}, \eta_{1} \in F \operatorname{Diff}(\Omega)$, and let

$$
X:=\left\{\eta \in C^{1}([0,1] ; F \operatorname{Diff}(\Omega)) \mid \eta(0)=\eta_{0}, \eta(1)=\eta_{1}\right\}
$$

Given a constant $\sigma \in \mathbb{R}^{+}, \bar{\rho}: \Omega \rightarrow \mathbb{R}^{+}$and $\varphi \in C^{1}\left(\mathbb{R}^{n}\right)$, critical points (if they exist) of the action $E: X \rightarrow \mathbb{R}$ via

$$
E(\eta)=\int_{0}^{1}\left(\int_{\Omega} \frac{\bar{\rho}}{2}\left|\partial_{t} \eta\right|^{2}-\varphi(\eta) d x-\int_{\Sigma(t)} \sigma d S\right) d t
$$

must satisfy the incompressible Euler equations with free boundary and surface tension

$$
\begin{cases}\rho\left(\partial_{t} u+u \cdot \nabla u\right)+\nabla p=-\nabla \varphi & \text { on } \Omega(t) \\ \operatorname{div} u=0 & \text { on } \Omega(t) \\ p=-\sigma H & \text { on } \Sigma(t)\end{cases}
$$

where $u: \Omega(t) \times[0,1] \rightarrow \mathbb{R}^{n}$ is the Eulerian velocity defined via $u(\eta(x, t), t)=\partial_{t} \eta(x, t)$,

$H=-\operatorname{div} \nu$ is the mean curvature of $\Sigma(t), \bar{\rho}(x)=\rho(\eta(x, t))$ is the density, and $p:[0,1] \rightarrow C^{\infty}(\Omega(t))$ is the pressure.

Finally, in the next two theorems we modify our action functionals to account for various surfactant dynamics, adding a second boundary term to penalize "wiggling" of surfactants along the boundary.

As part of our underlying assumptions, we enforce that the overall mass of the surfactants is locally (and hence globally) conserved. To be precise, let $J_{\Sigma}: \Sigma \times[0,1] \rightarrow \mathbb{R}$ be the distortion of the surface area element, so that for any $t \in[0,1], U \subseteq \Sigma$ and $f: \eta(U, t) \rightarrow \mathbb{R}^{n}$ smooth, then

$$
\int_{U} f \circ \eta(x, t) J_{\Sigma}(x, t) d S(x)=\int_{\eta(U, t)} f(x) d S(x) .
$$

Then setting $\gamma$ to be the Eulerian density of our surfactants and putting

$$
\bar{\gamma}(x, t) J_{\Sigma}(x, t)=\bar{\gamma}_{0}(x) \text {, and } \gamma(\eta(x, t), t)=\bar{\gamma}(x, t),
$$

our conservation of mass equation reads

$$
\frac{d}{d t} \int_{\eta(U, t)} \gamma d S=0
$$

for any $U \subseteq \Sigma$ measurable.

Now we first consider a model where the surfactants adhere to and remain stationary relative to the boundary, meaning that their movement is entirely dictated by $\eta$.

Theorem 1.7. Fix $\eta_{0}, \eta_{1} \in F \operatorname{Diff}(\Omega)$, let

$$
X=\left\{\eta \in C^{1}([0,1] ; F \operatorname{Diff}(\Omega)) \mid \eta(0)=\eta_{0}, \eta(1)=\eta_{1}\right\}
$$

Given $\bar{\rho}: \Omega \rightarrow \mathbb{R}^{+}, \xi: \mathbb{R} \rightarrow \mathbb{R}^{+}, \bar{\gamma}_{0}: \Sigma \rightarrow \mathbb{R}^{+}, \varphi \in C^{1}\left(\mathbb{R}^{n}\right)$, consider the action $E: X \rightarrow \mathbb{R}$ given by

$$
E(\eta)=\int_{0}^{1}\left(\int_{\Omega} \frac{\bar{\rho}}{2}\left|\partial_{t} \eta\right|^{2}-\varphi(\eta) d x+\int_{\Sigma} \frac{\bar{\gamma}_{0}}{2}\left|\partial_{t} \eta\right|^{2} d S-\int_{\Sigma(t)} \xi(\gamma) d S\right) d t
$$


with all relevant terms as defined in equations (1.9, 1.10, and 1.16). Then critical points (if they exist) of the action functional E must satisfy

$$
\begin{cases}\rho\left(\partial_{t} u+u \cdot \nabla u\right)+\nabla p=-\nabla \varphi & \text { on } \Omega(t) \\ \operatorname{div} u=0 & \text { on } \Omega(t) \\ \gamma\left(\partial_{t} u+u \cdot \nabla u\right)-p \nu=\nabla_{\Sigma(t)} \sigma+H \nu \sigma & \text { on } \Sigma(t) \\ \partial_{t} \gamma+\nabla \gamma \cdot u+\gamma \operatorname{div}_{\Sigma(t)} u=0 & \text { on } \Sigma(t)\end{cases}
$$

where again $u: \Omega(t) \times[0,1] \rightarrow \mathbb{R}^{n}$ is the Eulerian velocity defined via $u(\eta(x, t), t)=\partial_{t} \eta(x, t)$, $\sigma=\xi(\gamma)-\xi^{\prime}(\gamma) \gamma$ is the surface tension, $\bar{\rho}(x)=\rho(\eta(x, t), t)$, and $p:[0,1] \rightarrow C^{\infty}(\Omega(t))$.

Remark. Here $\xi$ is understood as the free energy on the boundary. For a detailed discussion of how and why surface tension $\sigma$ and free energy $\xi$ are related, see Equation (1.28) in [TW17]. In the case where the free energy $\xi$ is constant, it is the same as the surface tension $\sigma$, and hence was not considered separately in our previous results. In the simplest context, surface tension is constant. However, in many cases, surface tension decreases where there is a high concentration of surfactants and hence a larger surface tension is not penalized as much as it would otherwise be. $\xi$ takes this into account.

Remark. Note that the third equation of the results can be found in Equation (1.2) of [TW17].

Now, in the following theorem (Theorem 1.8), we will add an additional degree of freedom $\beta$ that allows the surfactants to slide freely along the boundary, with the associated motions then being generated by $\eta \circ \beta$. In particular, note that the surfactants on the boundary can now move in two ways. Firstly, as before, when the bulk moves, the surface itself can deform and cause the surfactants to move. Additionally, with the addition of $\beta$, the surfactants can now also move independently of the bulk along the surface. Our definitions then change slightly, so that we now require $J_{\Sigma}: \Sigma \times[0,1] \rightarrow \mathbb{R}$ to satisfy that

$$
\int_{U} f \circ \eta(t) \circ \beta(t) J_{\Sigma} d S=\int_{\eta(\beta(U, t), t)} f d S
$$

for any $U \subseteq \Sigma$ and $f: \eta(U, t) \rightarrow \mathbb{R}^{n}$ smooth and that

$$
\bar{\gamma}(x, t) J_{\Sigma}(x, t)=\bar{\gamma}_{0}(x), \gamma(\eta(\beta(x, t), t), t)=\bar{\gamma}(x, t) .
$$

As before, we will still enforce conservation of mass, so that for any $U \subseteq \Sigma$,

$$
\frac{d}{d t} \int_{\eta \circ \beta(U, t)} \gamma d S=0 .
$$

Remark. Note that although the results of Theorem 1.7 and Theorem 1.8 appear to be very similar, we emphasize that in Theorem 1.8, $u_{s}(\eta(\beta(x, t), t)):=\partial_{t}(\eta(\beta(x, t), t))$ is generated by both the motion of $\eta$ and by the motion of the surfactants $\beta$, whereas $u$ depends solely upon $\eta$.

Remark. As motivation for Theorem 1.8, we consider the following scenario. If we imagine that the initial volume $\Omega$ contains a droplet moving near the Earth's surface which is covered by a surfactant on its boundary, then $\beta$ describes the relative motion of the surfactant around the droplet with $u_{s}$ being the velocity of the surfactant around the bulk. For instance, the bulk can be rotating at some constant angular velocity while surfactant can be rotating at higher angular velocity. $\beta$ in this case accounts for relative difference between angular velocity of the bulk and the surfactant. The potential term $\varphi$ would then become the standard gravitational potential energy $\varphi:=\bar{\rho} g h$, where $g$ is the constant of gravitational acceleration and $h(\eta(x, t), t)$ is the distance between the surface of the Earth and the material point in question.

Theorem 1.8. Fix $\eta_{0}, \eta_{1} \in F \operatorname{Diff}(\Omega)$, let

$$
\begin{gathered}
X:=\left\{\eta \in C^{1}([0,1] ; \operatorname{FDiff}(\Omega)) \mid \eta(0)=\eta_{0}, \eta(1)=\eta_{1}\right\} \\
Y:=\left\{\beta \in C^{1}\left([0,1] ; \operatorname{Diff}_{0}(\Sigma)\right) \mid \beta(0, x)=x\right\} \\
\text { Given } \bar{\rho}: \Omega \rightarrow \mathbb{R}^{+}, \xi: \mathbb{R} \rightarrow \mathbb{R}^{+}, \bar{\gamma}_{0}: \Sigma \rightarrow \mathbb{R}^{+}, \varphi \in C^{1}\left(\mathbb{R}^{n}\right) \text {, consider } E: X \times Y \rightarrow \mathbb{R} \text { via } \\
E(\eta, \beta)=\int_{0}^{1}\left(\int_{\Omega} \frac{\bar{\rho}}{2}\left|\partial_{t} \eta\right|^{2}-\varphi(\eta) d x+\int_{\Sigma} \frac{\bar{\gamma}_{0}}{2}\left|\partial_{t}(\eta \circ \beta)\right|^{2} d S-\int_{\Sigma(t)} \xi(\gamma) d S\right) d t
\end{gathered}
$$


with all relevant terms as defined above.

Then critical points (if they exist) of the action functional E must satisfy

$$
\begin{cases}\rho\left(\partial_{t} u+u \cdot \nabla u\right)+\nabla p=-\nabla \varphi & \text { on } \Omega(t) \\ \operatorname{div} u=0 & \text { on } \Omega(t) \\ \gamma\left(\partial_{t} u_{s}+u_{s} \cdot \nabla u_{s}\right)-p \nu=\nabla_{\Sigma(t)} \sigma+H \nu \sigma & \text { on } \Sigma(t) \\ \partial_{t} \gamma+\nabla \gamma \cdot u+\gamma \operatorname{div}_{\Sigma(t)} u=0 & \text { on } \Sigma(t)\end{cases}
$$

where $u: \Omega(t) \times[0,1] \rightarrow \mathbb{R}^{n}$ is the Eulerian velocity of the fluid defined via $u(\eta(x, t), t)=\partial_{t} \eta(x, t)$, $u_{s}: \Sigma(t) \times[0,1] \rightarrow \mathbb{R}^{n}$ is the Eulerian velocity of the surfactant defined via $u_{s}(\eta(\beta(x, t), t), t)=\partial_{t}(\eta(\beta(x, t), t))$, $\sigma=\xi(\gamma)-\xi^{\prime}(\gamma) \gamma$ is the surface tension, $\bar{\rho}(x)=\rho(\eta(x, t), t)$ are densities, and $p:[0,1] \rightarrow C^{\infty}(\Omega)$ is the pressure.

Examining the equations given by (1.23), we see that the first two govern the motion of the incompressible fluid, whereas the last two describe the evolution of the surfactants living on the boundary. In particular, the third equation describes the kinematics of surfactants whereas the fourth enforces preservation of surfactant mass on the boundary.

\section{Technical Prerequisites}

We'll now present some technical results that will be used heavily in the derivations of our final PDEs. Beginning with some standard results from fluid mechanics, in Sections 2.1 and 2.2, we revisit the relevant function spaces we'll be working in, taking care to establish the different structural properties that we'll be taking advantage of. Of particular interest from these sections is Lemma 2.5, which allows us to perturb any given flow map by an arbitrary divergence-free velocity field, an argument that provides us with explicit constraints on the action critical points being considered.

Supplementing these structural results are then the decompositions of $L^{2}$ provided by Section 2.3, which allow us to introduce the pressure by way of complementing particular classes of divergence free velocity fields in $L^{2}$.

Finally, we conclude by presenting some useful computation tools from differential geometry in Section 2.4.

\subsection{Basic Fluid Mechanics.}

We first show how volume preserving flows are related to divergence free velocity fields.

Definition 2.1. Let $\Omega \subseteq \mathbb{R}^{n}$ be open and $f: \Omega \rightarrow f(\Omega) \subseteq \mathbb{R}^{n}$ be a $C^{1}$ diffeomorphism. We say $f$ is volume preserving if $\mu_{n}(U)=\mu_{n}(f(U))$ for any measurable $U \subseteq \Omega$, where $\mu_{n}$ is the $n$-dimensional Lebesgue measure.

Lemma 2.2. Let $\eta: \Omega \times[0,1] \rightarrow \mathbb{R}^{n}$ be a flow map and write $\Omega(t)=\eta(\Omega, t)$. If $u(\cdot, t): \Omega(t) \rightarrow \mathbb{R}^{n}$ is the Eulerian velocity associated to $\eta$, then

$$
\partial_{t} \operatorname{det} D \eta(x, t)=\operatorname{div} u(\eta(x, t), t) \operatorname{det} D \eta(x, t)
$$

Proof. This proof is not new (see, for instance, [BF13]) but is being included for convenience. We begin by recalling Jacobi's Formula. Given a $C^{1}$ map $A: \mathbb{R} \rightarrow \mathbb{R}^{m \times m}$, we have that

$$
\frac{d}{d t} \operatorname{det} A(t)=\operatorname{tr}\left(\operatorname{adj}(A(t)) \frac{d A(t)}{d t}\right)
$$

where $\operatorname{adj}(A(t))$ is the adjugate of $A(t)$. 
Our desired result is then an easy calculation using chain rule, the definition of the adjugate, and the stated equation. By direct computation,

$$
\begin{aligned}
\partial_{t} \operatorname{det} D \eta(x, t) & =\operatorname{tr}\left(\operatorname{adj}(D \eta(x, t)) \partial_{t} D \eta(x, t)\right) \\
& =\operatorname{tr}(\operatorname{adj}(D \eta(x, t)) D u(\eta(x, t), t) D \eta(x, t)) \\
& =\operatorname{tr}(D u(\eta(x, t), t) D \eta(x, t) \operatorname{adj}(D \eta(x, t))) \\
& =\operatorname{tr}(D u(\eta(x, t), t) I \operatorname{det} D \eta(x, t)) \\
& =\operatorname{tr}(D u(\eta(x, t), t)) \operatorname{det} D \eta(x, t) \\
& =\operatorname{div} u(\eta(x, t), t) \operatorname{det} D \eta(x, t) .
\end{aligned}
$$

Theorem 2.3. Let $\eta: \Omega \times[0,1] \rightarrow \mathbb{R}^{n}$ be a flow map and write $\Omega(t)=\eta(\Omega, t)$. Then the following are equivalent:

(1) For all $t \in[0,1]$ the map $\eta(\cdot, t): \Omega \rightarrow \Omega(t)$ is volume preserving.

(2) $\operatorname{det} D \eta(x, t)=1$ for all $x \in \Omega, t \in[0,1]$.

(3) If $u(\cdot, t): \Omega(t) \rightarrow \mathbb{R}^{n}$ is the Eulerian velocity associated to $\eta$, i.e., $u(\eta(x, t), t)=\partial_{t} \eta(x, t)$, then $\operatorname{div} u(x, t)=0$ for all $x \in \Omega(t)$ and $t \in[0,1]$.

Proof. We first show the equivalence of the first two items.

If $U \subseteq \Omega$ is measurable, then $U(t)=\eta(U, t)$ is measurable and by change of variables with $\eta(t)$ we have

$$
\mu_{n}(U(t))=\int_{\eta(U, t)} 1 d x=\int_{U} \operatorname{det} D \eta(x, t) d x .
$$

Hence if $\mu_{n}(U)=\mu_{n}(U(t))$ for all such $U$ measurable, then

$$
\int_{U}[\operatorname{det} D \eta(x, t)-1] d x=0
$$

and so $\operatorname{det} D \eta(x, t)=1$ for all $x \in \Omega, t \in[0,1]$. The converse is immediate.

Now suppose the second item. Then by Lemma 2.2,

$$
\begin{aligned}
\operatorname{det} D \eta(x, t)=1 \Longrightarrow 0 & =\partial_{t} \operatorname{det} D \eta(x, t) \\
& =\operatorname{div} u(\eta(x, t), t) \operatorname{det} D \eta(x, t)=\operatorname{div} u(\eta(x, t), t)
\end{aligned}
$$

for all $x \in \Omega, t \in[0,1]$. Since $\eta(\cdot, t): \Omega \rightarrow \Omega(t)$ is a diffeomorphism, div $u(x, t)=0$ for all $t \in[0,1], x \in \Omega(t)$. Finally, suppose the third item. Then

$$
\left\{\begin{array}{l}
\partial_{t} \operatorname{det} D \eta(x, t)=\operatorname{div} u(\eta(x, t), t) \operatorname{det} D \eta(x, t)=0 \\
\operatorname{det} D \eta(x, 0)=1
\end{array}\right.
$$

and thus det $D \eta(x, t)=1$ for all $x \in \Omega, t \in[0,1]$.

\subsection{The Structure of $\operatorname{Diff}_{0}(\Omega)$ and FDiff $(\Omega)$.}

With these prerequisites out of the way, we're now ready to introduce our function spaces and their properties. Thinking of $\operatorname{Diff}_{0}(\Omega)$ and $\operatorname{FDiff}(\Omega)$ as manifolds in $L^{2}\left(\Omega ; \mathbb{R}^{n}\right)$ (which is the result of the following theorem), we can formally compute the tangent spaces at any function $\eta \in \operatorname{FDiff}(\Omega)$. Using these characterizations, we can then reason about variational properties of paths along these manifolds.

Theorem 2.4. FDiff $(\Omega)$ and Diff $(\Omega) \subseteq L^{2}\left(\Omega ; \mathbb{R}^{n}\right)$ are Fréchet manifolds, with their respective tangent spaces at any point $\eta$ satisfying

$$
\begin{aligned}
T_{\eta} \operatorname{Diff}_{0}(\Omega) & =\left\{u \circ \eta \in C^{\infty}\left(\Omega ; \mathbb{R}^{n}\right) \mid \operatorname{div} u=0, u \cdot \nu=0\right\} \\
T_{\eta} F \operatorname{Diff}(\Omega) & =\left\{u \circ \eta \in C^{\infty}\left(\Omega ; \mathbb{R}^{n}\right) \mid \operatorname{div} u=0\right\}
\end{aligned}
$$

Proof. For the proof that $\operatorname{Diff}_{0}(\Omega)$ is a manifold, see section VIII.1 of [Omo74] or [Sch87].

For $\operatorname{FDiff}(\Omega)$, we simply modify the proof slightly and see that every volume element on $\Omega$ is the restriction 
of some volume form on $\mathbb{R}^{n}$ to $\Omega$.

We now show $\subseteq$ in 2.1 for the $\operatorname{Diff}_{0}(\Omega)$ case. First recall that

$$
T_{\eta} \operatorname{Diff}_{0}(\Omega)=\left\{\gamma^{\prime}(0) \mid \gamma \in C^{\infty}\left((-\varepsilon, \varepsilon) ; \operatorname{Diff}_{0}(\Omega)\right), \gamma(0)=\eta\right\}
$$

and note that any such curve $\gamma$ as above can be lifted to a $C^{\infty}$ volume-preserving flow map $\Gamma:(-\varepsilon, \varepsilon) \times \Omega \rightarrow$ $\Omega$ via $\Gamma(t, x)=\gamma(t)(x)$. It's obvious that $\partial_{t} \Gamma=\gamma^{\prime}$ if the latter exists, so we have

$$
T_{\eta} \operatorname{Diff}_{0}=\left\{\partial_{t} \Gamma(0, \cdot) \mid \Gamma \in C^{1}(\mathbb{R} \times \Omega ; \Omega), \Gamma(0, \cdot)=\eta \text { and } \forall t \operatorname{div} \partial_{t} \Gamma(t, \cdot)=0, \partial_{t} \Gamma \cdot \nu=0\right\}
$$

where the first condition follows from the fact that $\Gamma$ is volume preserving and last condition follows from the fact that $\Gamma(t, \cdot) \in \operatorname{Diff}_{0}$. This clearly shows $\subseteq$.

We now show $\supseteq$.

Suppose that $u \in C^{\infty}\left(\Omega ; \mathbb{R}^{n}\right)$ satisfies the conditions of Equation (2.1). It suffices to show that there exists $\varepsilon>0$ and $\Gamma \in C^{\infty}((-\varepsilon, \varepsilon) \times \Omega ; \Omega)$ such that $\partial_{t} \Gamma(0, \cdot)=u$. To do so, we reframe this as solving the ODE

$$
\left\{\begin{array}{lc}
\partial_{t} \Gamma(t, x)=u(\Gamma(t, x)) & x \in \Omega, t \in(-\varepsilon, \varepsilon) \\
\Gamma(0, x)=\eta(x) & x \in \Omega .
\end{array}\right.
$$

Then smoothness of the initial data and $u$ allow us to construct $\Gamma \in C^{\infty}\left((-\varepsilon, \varepsilon) \times \Omega ; \mathbb{R}^{n}\right)$ solving this system. The fact that the normal component of $v$ along the boundary vanishes then guarantees that $\operatorname{im}(\Gamma) \subseteq \Omega$. Bijectivity of every such $\Gamma(t, \cdot)$ follows from the fact that $v$ is divergence free and thus $\partial_{t} \Gamma$ is also divergence free, so such functions are volume-preserving and thus bijective.

The proof of Equation (2.2) follows similarly. Without the condition that $u \cdot \nu=0$, the fixed boundary is no longer enforced.

Given the above characterization of these tangent spaces, a natural question to ask is if a path along these manifolds can be perturbed by a tangent-space-valued velocity field. The following lemma answers this question affirmatively, showing that for arbitrary divergence free velocity fields and paths $\eta$, there exists a perturbation of $\eta$ (denoted by $\zeta$ ) such the derivative of $\zeta$ at $\eta$ is equal to the desired velocity field.

Combined with the decompositions of Section 2.3, this result will be crucial in obtaining many of the optimality conditions we derive later.

Lemma 2.5. Let $v_{0}:[0,1] \rightarrow\left\{v \in C^{\infty}\left(\Omega ; \mathbb{R}^{n}\right) \mid \operatorname{div}\left(v \circ \eta^{-1}\right)=0\right\}$ be a smooth velocity field with $v_{0}(0)=v_{0}(1)=0$, and fix $\eta \in C^{1}([0,1], F D i f f(\Omega))$. Set also

$$
\eta_{0}:=\eta(0), \eta_{1}:=\eta(1) \in F \operatorname{Fiff}(\Omega)
$$

Then there exists $\varepsilon>0$ and a perturbation $\zeta(s) \in C^{\infty}\left((-\varepsilon, \varepsilon) ;\left\{\eta:[0,1] \rightarrow F \operatorname{Diff}(\Omega) \mid \eta(0)=\eta_{0}, \eta(1)=\eta_{1}\right\}\right)$ such that $\zeta(0)=\eta$ and $\partial_{s} \zeta(x, 0, t)=v_{0}(\eta(x, t), t)$.

Proof. Consider the following ODE:

$$
\left\{\begin{array}{l}
v(0)=v(1)=0 \\
\partial_{s} \varphi(x, s, t)=v(\varphi(x, s, t), s, t) \\
\varphi(x, 0, t)=x
\end{array}\right.
$$

where we define $v(\eta(x, t), s, t)=v_{0}(\eta(x, t), t)$. This is a flow map ODE associated to $v$, so we can apply the Cauchy-Peano Theorem to produce such a solution $\varphi$.

If we let $\zeta(x, s, t)=\varphi(\eta(x, t), s, t)$, then clearly $\zeta(0)=\eta$ by the third condition. We also see that

$$
\partial_{s} \zeta(x, 0, t)=v(\varphi(\zeta(x, 0, t), 0, t), 0, t)=v(\zeta(x, 0, t), 0, t)
$$

which is exactly what we wanted. In order to check that $\zeta(s) \in C^{\infty}$, we can reframe our ODE slightly by writing $s$ as a function of $t$, so that $s(t) \equiv s$ for any fixed $s$, so that $s$ becomes part of the initial data. Our updated ODE becomes:

$$
\left\{\begin{array}{l}
v(0)=v(1)=0 \\
\partial_{s} \varphi(x, s(t), t)=v(\varphi(x, s(t), t), s(t), t) \\
\varphi(x, 0, t)=x \\
s^{\prime}(t)=0, s(0)=s
\end{array}\right.
$$


so in either case we see that $\zeta(x, s, t):=\varphi(\eta(x, t), s, t)$ satisfies the requirements.

\subsection{The Structure of $L^{2}$.}

We now derive two useful decompositions of $L^{2}$ that characterize the orthogonal complement of certain divergence free vector fields.

The first decomposition is the well-known Leray decomposition, which we state as follows.

Theorem 2.6. (Leray Decomposition)

Let

$$
\mathcal{V}=\left\{\varphi \in C_{c}^{\infty}\left(\Omega ; \mathbb{R}^{n}\right) \mid \operatorname{div} \varphi=0\right\}
$$

Let $H$ be the closure of $\mathcal{V}$ in $L^{2}\left(\Omega ; \mathbb{R}^{n}\right)$. Then

$$
H=\left\{u \in L^{2}\left(\Omega ; \mathbb{R}^{n}\right) \mid \operatorname{div} u=0, u \cdot \nu=0\right\}
$$

and the orthogonal complement of $H$ in $L^{2}\left(\Omega ; \mathbb{R}^{n}\right)$ satisfies

$$
H^{\perp}=\left\{\nabla p \in L^{2}\left(\Omega ; \mathbb{R}^{n}\right) \mid p \in H^{1}(\Omega)\right\}
$$

Proof. See Theorem IV.3.5 of [BF13].

Remark. Here the assertion that $u \cdot \nu=0$ on $\Sigma$ (and $p=0$ in the next theorem) should not be confused with a pointwise result, since the value of an $L^{2}$ function on a null set is not well-defined. It is understood by first considering the operator $\gamma_{\nu}(u)=u \cdot \nu$ on $C^{1}\left(\bar{\Omega} ; \mathbb{R}^{n}\right)$ and then extending it by linearity to all of $L^{2}\left(\Omega ; \mathbb{R}^{n}\right)$. Additionally, the divergence operator here should be understood in the sense of distributions, meaning that it can operate on arbitrary functions in $L^{2}$ without any additional assumptions on smoothness.

Theorem 2.7. (A different decomposition of $L^{2}$ )

Let

$$
H^{\prime}=\left\{u \in L^{2}\left(\Omega ; \mathbb{R}^{n}\right) \mid \operatorname{div} u=0\right\}
$$

Then $H^{\prime}$ is a closed subspace of $L^{2}\left(\Omega ; \mathbb{R}^{n}\right)$ and the orthogonal complement of $H^{\prime}$ in $L^{2}\left(\Omega ; \mathbb{R}^{n}\right)$ is

$$
H^{\prime \perp}=\left\{\nabla p \in L^{2}\left(\Omega ; \mathbb{R}^{n}\right) \mid p \in H^{1}(\Omega), p=0 \text { on } \Sigma\right\}
$$

Proof. The fact $H^{\prime}$ is closed is immediate from continuity of the divergence operator, so it suffices to show the equality.

Towards doing so, we first write

$$
X=\left\{\nabla p \in L^{2}\left(\Omega ; \mathbb{R}^{n}\right) \mid p \in H^{1}(\Omega), p=0 \text { on } \Sigma\right\}
$$

Now let $u \in X$; then for all $v \in H^{\prime}$ we have

$$
(u, v)_{L^{2}}=\int_{\Omega} u \cdot v d x=\int_{\Omega} \nabla p \cdot v d x=\int_{\partial \Omega} p v \cdot \nu-\int_{\Omega} p \operatorname{div} v d x=0
$$

This obviously shows that $X \subseteq H^{\prime \perp}$.

Conversely, suppose $u \in H^{\prime \perp}$. Then since $H^{\prime} \supseteq H$, we know that $H^{\prime \perp} \subseteq H^{\perp}$, and so by Theorem 2.6, we know that $u=\nabla p$ for some $p$. Now given any $\psi \in C^{\infty}\left(\mathbb{R}^{n}\right)$, we set $\tilde{\psi}=\psi-f_{\partial \Omega} \psi$, where $f_{\Sigma} \psi=\frac{1}{|\Sigma|} \int_{\Sigma}$ is the average of $\psi$ on $\Sigma$, and consider integrating $u$ against $v=\nabla \varphi$, where $\varphi$ is the solution to the Laplace-Neumann equation

$$
\left\{\begin{array}{l}
\Delta \varphi=0 \text { on } \Omega \\
\partial_{\nu} \varphi=\tilde{\psi} \text { on } \Sigma .
\end{array}\right.
$$

In doing so, we note in particular that such $\varphi$ always exist since $\int_{\partial \Omega} \tilde{\psi}=0$ and also that the first condition suffices to show that $v \in H^{\prime}$. 
Now we note that we have

$$
\begin{aligned}
\int_{\Sigma} p \tilde{\psi} & =\int_{\Sigma} p v \cdot \nu=\int_{\Omega} \operatorname{div}(p v) \\
& =\int_{\Omega} \nabla p \cdot v+p \operatorname{div} v \\
& =\int_{\Omega} u v+\int_{\Omega} p \operatorname{div} v \\
& =0
\end{aligned}
$$

and also that

$$
\begin{aligned}
0=\int_{\Sigma} p \tilde{\psi} & =\int_{\Sigma} p \psi-\frac{1}{|\Sigma|}\left(\int_{\Sigma} p\right)\left(\int_{\Sigma}\right) \\
& =\int_{\Sigma} p \psi-\int_{\Sigma}\left(f_{\Sigma} p\right) \\
& =\int_{\Sigma}\left(p-f_{\Sigma} p\right)
\end{aligned}
$$

Now since $\psi$ was chosen arbitrarily, we thus conclude that $p=f_{\Sigma} p$ on $\Sigma$, which means that $p$ is constant on $\Sigma$. Setting $\tilde{p}=p-f_{\Sigma} p$, we find that $u=\nabla \tilde{p}$, where $\tilde{p}=0$ on $\Sigma$ as desired.

\subsection{Calculus on Hypersurfaces.}

In this section we present a few vital theorems on calculus over hypersurfaces. Later, we'll use the Reynold's Transport Theorem on hypersurfaces to perturb the flow map with respect to $s$ rather than with respect to $t$. We'll then use the surface divergence theorem to simplify some terms appearing as a consequence of Reynold's Transport Theorem.

Finally, we introduce a lemma that characterizes functions that vanish when tested against arbitrary divergence free.

Definition 2.8. Given a $C^{2}$ hypersurface $\Sigma \subseteq \mathbb{R}^{n}$ with $\nu: \Sigma \rightarrow \mathbb{R}^{n}$ the outward pointing unit normal, $f \in C^{1}(\Sigma ; \mathbb{R})$ and $X \in C^{1}\left(\Sigma ; \mathbb{R}^{n}\right)$, we define the surface gradient of $f$ as

$$
\nabla_{\Sigma} f=(I-\nu \otimes \nu) \nabla f
$$

and the surface divergence of $X$ as

$$
\operatorname{div}_{\Sigma} X=\operatorname{tr}((I-\nu \otimes \nu) D X) .
$$

Given the definition of the surface divergence, we have an analog of the traditional divergence theorem but for surfaces.

Theorem 2.9. (Surface Divergence Theorem)

Let $\Sigma, f$, and $X$ be as above. Then

$$
\int_{\Sigma} f \operatorname{div}_{\Sigma} X d S=-\int_{\Sigma} \nabla_{\Sigma} f \cdot X+H X \cdot \nu f d S
$$

where $H=-\operatorname{div} \nu$ is the mean curvature.

Proof. See Theorem 3.2.1 and Remark 4 of [LY02]. Their result appears superficially different, but after correcting notational differences and by considering the vector field $X^{\prime}:=f X$, this form of the surface divergence theorem is simply a result of the product rule.

Similarly, we have a version of the Reynolds Transport Theorem, which will be used to differentiate functionals in later calculations. 
Theorem 2.10. (Reynolds Transport Theorem on Hypersurfaces)

Let $\Sigma$ be as above and $\beta \in C^{1}\left(\Sigma \times[0,1] ; \mathbb{R}^{n}\right)$. Set $\Sigma(t)=\beta(\Sigma, t)$.

If $f \in C^{1}\left(\mathbb{R}^{n} \times[0,1] ; \mathbb{R}^{n}\right)$, then

$$
\frac{d}{d t} \int_{\Sigma(t)} f d S=\int_{\Sigma(t)} \partial_{t} f+\nabla f \cdot u+f \operatorname{div}_{\Sigma(t)} u d S
$$

where $u(\beta(x, t), t)=\partial_{t} \beta(x, t)$.

Proof. See equations (2) and (4) of [Sto90] or page 5 of [HLT08] or Equation (3.4-19) of [EBW91].

Finally, the following lemma will be relevant when we consider integration of a particular smooth function defined on $\Sigma$ against arbitrary divergence free test functions. If such an integral is always zero, then this lemma allows us to conclude that the smooth function is some constant multiple of the outward-pointing normal.

Lemma 2.11. Let $\Sigma=\partial \Omega \subseteq \mathbb{R}^{n}$ be a smooth hypersurface, $\nu$ be it's outward pointing normal, and $X \in C^{\infty}\left(\Sigma ; \mathbb{R}^{n}\right)$.

Suppose that for all divergence free $v \in C^{1}\left(\bar{\Omega} ; \mathbb{R}^{n}\right)$ we have

$$
\int_{\Sigma} X \cdot v d S=0
$$

Then $X=c_{0} \nu$ for some constant $c_{0} \in \mathbb{R}$.

Proof. We begin by noting that we can decompose $X=X_{\nu}+X_{\|}$, where $X_{\nu}$ and $X_{\|}$are the normal and tangential components of $X$ respectively. Then $X_{\|}$is tangent to $\Sigma$, and hence by Lemma 3.5.5 in [Sch95], we can extend $X_{\|}$onto the interior of $\Omega$. In particular, there must exist $v$ which is smooth and divergence free on $\Omega$ and defined on $\bar{\Omega}$ such that $v=X_{\|}$on $\Sigma$. By hypothesis, we have that

$$
0=\int_{\Sigma} X \cdot v d S
$$

so using our decomposition and noting that $X_{\|} \cdot X_{\nu}=0$, we find that

$$
\begin{aligned}
0 & =\int_{\Sigma}\left(X_{\nu}+X_{\|}\right) \cdot v d S \\
& =\int_{\Sigma} X_{\nu} \cdot X_{\|} d S+\int_{\Sigma} X_{\|} \cdot X_{\|} d S \\
& =\int_{\Sigma}\left\|X_{\|}\right\|^{2} d S
\end{aligned}
$$

Of course, this implies that $X_{\|}=0$ on $\Sigma$, and hence $X$ has no tangential component. In particular, there exists a smooth $f: \Sigma \rightarrow \mathbb{R}$ such that $X=f \nu$.

We claim now that $f$ is constant. To see this, consider any mean-zero function $g$ defined on $\Sigma$. By solving Laplace's equation, we can find $\varphi: \bar{\Omega} \rightarrow \mathbb{R}$ with $\Delta \varphi=0$ on $\Omega$, and $\varphi=g_{\nu}$ (the normal derivative) on $\Sigma$. Then, define $v=\nabla \varphi$. Since $\operatorname{div} v=\Delta \varphi=0$, we must have that

$$
\begin{aligned}
0=\int_{\Sigma} X \cdot v d S=\int_{\Sigma} f \nu \cdot \nabla \varphi d S & =\int_{\Sigma} f \varphi_{\nu} d S \\
& =\int_{\Sigma} f g d S
\end{aligned}
$$

In particular, for any mean-zero function $g$ defined on $\Sigma$, we have that $\int_{\Sigma} f g d S=0$ which allows us to immediately conclude that $f=c_{0}$ for some $c_{0} \in \mathbb{R}$. Thus, $X=c_{0} \nu$ as desired. 


\section{Proofs of Main Results}

We now prove the main results as stated in the introduction, beginning with Arnold's formulation of the Euler equation.

Proof of Theorem 1.4. Fix any $v:[0,1] \rightarrow\left\{u \in L^{2}\left(\Omega ; \mathbb{R}^{n}\right) \mid \operatorname{div} u=0, u \cdot \nu=0\right\}$, and using Lemma 2.5, set $\zeta(s) \in C^{\infty}((-\varepsilon, \varepsilon) ; X)$ to be such that $\left.\partial_{s}\right|_{s=0} \zeta=v \circ \eta$.

Using the fact that $E(\zeta)$ must achieve a minimum at $s=0$, we then find that by Fubini,

$$
0=\left.\partial_{s}\right|_{s=0} E(\zeta)=\int_{0}^{1} \int_{\Omega} \partial_{t} \eta \cdot \partial_{t} \partial_{s} \zeta d x d t=\int_{\Omega} \int_{0}^{1} \partial_{t} \eta \cdot \partial_{t}(v \circ \eta) d t d x
$$

Integrating by parts in time (and using the fact that $v$ vanishes when $t=0,1$ ), this expression then just becomes

$$
-\int_{\Omega} \int_{0}^{1} \partial_{t}^{2} \eta \cdot(v \circ \eta) d t d x
$$

which, upon using Fubini and change of variables with $\eta(t)$, yields

$$
-\int_{0}^{1} \int_{\Omega(t)} \partial_{t}^{2} \eta \circ \eta(t)^{-1} \cdot v d x d t=-\int_{0}^{1} \int_{\Omega(t)}\left(\partial_{t} u+u \cdot \nabla u\right) \cdot v d x d t=0
$$

where $u(\eta(x, t), t):=\partial_{t} \eta(x, t)$ is the Eulerian velocity of the flow $\eta$. Note that because $\partial_{t} \eta(x, t)=$ $\partial_{t} u(\eta(x, t), t)+u(\eta(x, t), t) \cdot \nabla u(\eta(x, t), t)$, we have the substitution $\partial_{t}^{2} \eta \circ \eta^{-1}=\partial_{t} u+u \cdot \nabla u$. This gives us the last equality.

Since $v$ was chosen arbitrarily, we may again use the Lebesgue differentiation theorem in time and the Leray Decomposition (Theorem 2.6) to find that

$$
\partial_{t} u+u \cdot \nabla u+\nabla p=0
$$

for some $p:[0,1] \rightarrow H^{1}(\Omega)$.

Then using the fact that $\eta$ is a volume-preserving diffeomorphism of $\Omega$ onto itself and Theorem 2.3, we derive the full set of equations

as desired.

$$
\begin{cases}\partial_{t} u+u \cdot \nabla u+\nabla p=0 & \text { on } \Omega \\ \operatorname{div} u=0 & \text { on } \Omega \\ u \cdot \nu=0 & \text { on } \Sigma\end{cases}
$$

We now prove Theorem 1.5, which is a modification to the original Arnold action functional that includes a globally defined potential energy term $\varphi$.

Proof of Theorem 1.5. Similar to the proof of the Arnold equation, we have

$$
\begin{aligned}
\left.\partial_{s}\right|_{s=0} E(\zeta) & =\int_{0}^{1} \int_{\Omega} \bar{\rho} \partial_{t} \eta \cdot \partial_{t} \partial_{s} \zeta-\nabla \varphi \cdot \partial_{s} \zeta d x d t=\int_{\Omega} \int_{0}^{1} \bar{\rho} \partial_{t} \eta \cdot \partial_{t}(v \circ \eta)-\nabla \varphi \cdot v d t d x \\
& =-\int_{\Omega} \int_{0}^{1} \bar{\rho} \partial_{t}^{2} \eta \cdot(v \circ \eta)+\nabla \varphi \cdot v d t d x=-\int_{0}^{1} \int_{\Omega(t)}\left(\left(\bar{\rho} \partial_{t}^{2} \eta\right) \circ \eta(t)^{-1}+\nabla \varphi\right) \cdot v d x d t
\end{aligned}
$$

Now defining $\rho(x, t)=\bar{\rho}\left(\eta^{-1}(x, t)\right)$ and using the identity $\partial_{t}^{2} \eta \circ \eta(t)^{-1}=\partial_{t} u+u \cdot \nabla u$, we substitute into the above to find

$$
\left.\partial_{s}\right|_{s=0} E_{1}(\zeta)=-\int_{0}^{1} \int_{\Omega(t)}\left(\rho\left(\partial_{t} u+u \cdot \nabla u\right)+\nabla \varphi\right) \cdot v d x d t
$$

which similarly implies that

$$
\int_{\Omega(t)}\left(\rho\left(\partial_{t} u+u \cdot \nabla u\right)+\nabla \varphi\right) \cdot v(t) d x=0 \text { for any divergence free } v(t) \in C^{\infty}\left(\Omega(t) ; \mathbb{R}^{n}\right)
$$

Using Theorem 2.7, this allows us to find $p:[0,1] \rightarrow H^{1}(\Omega)$ such that

$$
\rho\left(\partial_{t} u+u \cdot \nabla u\right)+\nabla \varphi+\nabla p=0 .
$$


Combining these results yields that

$$
\begin{cases}\rho\left(\partial_{t} u+u \cdot \nabla u\right)+\nabla p=-\nabla \varphi & \text { on } \Omega(t) \\ \operatorname{div} u=0 & \text { on } \Omega(t) \\ p=0 & \text { on } \Sigma(t)\end{cases}
$$

as desired.

We now prove Theorem 1.6.

Proof of Theorem 1.6. Similarly to the previous proof, we first split the action into

$$
E_{1}(\eta):=\int_{0}^{1} \int_{\Omega} \frac{\bar{\rho}}{2}\left|\partial_{t} \eta\right|^{2}-\varphi(\eta) d x d t \quad \text { and } \quad E_{2}(\eta):=\int_{0}^{1} \int_{\Sigma(t)} \sigma d S d t
$$

and consider $v, \zeta$ as before. We first isolate the contribution of $E_{1}$ by restriction of $\zeta$ to perturbations that are only compactly supported, meaning that $\partial_{s} \zeta^{\prime}=v \circ \eta$ for $v(t) \in C_{c}^{\infty}\left(\Omega(t) ; \mathbb{R}^{n}\right)$, a condition that suffices to guarantee $\partial_{s} E_{2}(\zeta)=0$ since the values along the boundary remain fixed. Then exactly as in Theorem 1.5 , we find that

$$
\left.\partial_{s}\right|_{s=0} E_{1}(\zeta)=-\int_{0}^{1} \int_{\Omega(t)}\left(\rho\left(\partial_{t} u+u \cdot \nabla u\right)+\nabla \varphi\right) \cdot v
$$

which means that

$$
\int_{\Omega(t)}\left(\rho\left(\partial_{t} u+u \cdot \nabla u\right)+\nabla \varphi\right) \cdot v(t) d x=0 \text { for any divergence free } v(t) \in C_{c}^{\infty}\left(\Omega(t) ; \mathbb{R}^{n}\right) .
$$

Then using the Leray decomposition from Theorem 2.6 again, we have

$$
\rho\left(\partial_{t} u+u \cdot \nabla u\right)+\nabla \varphi+\nabla \tilde{p}=0
$$

for some $\tilde{p}:[0,1] \rightarrow H^{1}(\Omega)$. Using this equality and the divergence theorem, we can then compute variations of both energies as

$$
\begin{aligned}
& \left.\partial_{s}\right|_{s=0} E_{1}(\zeta)=\int_{0}^{1} \int_{\Omega(t)} \nabla \tilde{p} \cdot v d x d t=\int_{0}^{1} \int_{\Sigma(t)} \tilde{p} \nu \cdot v d S d t \\
& \left.\partial_{s}\right|_{s=0} E_{2}(\zeta)=\partial_{s} \int_{0}^{1} \int_{\partial \zeta(\Omega, t, s)} \sigma d S d t=\int_{0}^{1} \int_{\Sigma(t)} \sigma \operatorname{div}_{\Sigma(t)} v d S d t
\end{aligned}
$$

where in the second equation we use the Reynolds Transport equation on Hypersurfaces (Theorem 2.10). Continuing the second calculation by using the surface divergence theorem (Theorem 2.9), we then find the variation of $E_{2}$ is

$$
-\int_{0}^{1} \int_{\Sigma(t)} \nabla_{\Sigma(t)} \sigma \cdot v+H v \cdot \nu \sigma d S d t=-\int_{0}^{1} \int_{\Sigma(t)} \sigma H v \cdot \nu d S d t
$$

Combining these two terms, we then find that for any divergence free $v$ we have that

$$
\begin{aligned}
0=\left.\partial_{s}\right|_{s=0} E(\zeta) & =\int_{0}^{1} \int_{\Sigma(t)} \tilde{p} \nu \cdot v d S d t+\int_{0}^{1} \int_{\Sigma(t)} \sigma H v \cdot \nu d S d t \\
& =\int_{0}^{1} \int_{\Sigma(t)}(\tilde{p} \nu+\sigma H \nu) \cdot v d S d t
\end{aligned}
$$

Thus by Lemma 2.11, for some constant $c_{0} \in \mathbb{R}^{+}$,

$$
\tilde{p} \nu+\sigma H \nu=c_{0} \nu \text { on } \Sigma(t)
$$

Defining $p:=\tilde{p}-c_{0}$, we derive

$$
\begin{cases}\rho\left(\partial_{t} u+u \cdot \nabla u\right)+\nabla p=-\nabla \varphi & \text { on } \Omega(t) \\ \operatorname{div} u=0 & \text { on } \Omega(t) \\ p=-\sigma H & \text { on } \Sigma(t) .\end{cases}
$$


We now prove Theorem 1.7.

Proof of Theorem 1.7. We first write $E$ as $E_{1}+E_{2}-E_{3}$ where

$$
\begin{aligned}
& E_{1}(\eta):=\int_{0}^{1} \int_{\Omega} \frac{\bar{\rho}}{2}\left|\partial_{t} \eta\right|^{2}-\varphi(\eta) d x d t \\
& E_{2}(\eta):=\int_{0}^{1} \int_{\Sigma} \frac{\bar{\gamma}_{0}}{2}\left|\partial_{t} \eta\right|^{2} d S d t \\
& E_{3}(\eta):=\int_{0}^{1} \int_{\Sigma(t)} \xi(\gamma) d S d t
\end{aligned}
$$

and consider $v, \zeta$ as before.

Again restricting to compactly supported $\zeta$ as before to isolate the contribution of $E_{1}$, we find that as in Theorem 1.5, there exists $\tilde{p}:[0,1] \rightarrow H^{1}(\Omega)$ such that

$$
\rho\left(\partial_{t} u+u \cdot \nabla u\right)+\nabla \varphi+\nabla \tilde{p}=0
$$

as before. Making this substitution, we now turn our attention back to general $\zeta$. Using the divergence theorem, we first calculate that

$$
\left.\partial_{s}\right|_{s=0} E_{1}(\zeta)=\int_{0}^{1} \int_{\Omega(t)} \nabla \tilde{p} \cdot v d x d t=\int_{0}^{1} \int_{\Sigma(t)} \tilde{p} \nu \cdot v d S d t .
$$

Then the same calculation along with an integration by parts yields

$$
\begin{aligned}
\left.\partial_{s}\right|_{s=0} E_{2}(\zeta) & =\int_{0}^{1} \int_{\Sigma} \bar{\gamma}_{0} \partial_{t} \eta \cdot \partial_{t} \partial_{s} \zeta d S d t \\
& =\int_{0}^{1} \int_{\Sigma} \bar{\gamma}_{0} \partial_{t} \eta \cdot \partial_{t}(v \circ \eta) d S d t=-\int_{0}^{1} \int_{\Sigma} \bar{\gamma}_{0} \partial_{t}^{2} \eta \cdot(v \circ \eta) d S d t
\end{aligned}
$$

Using the identity $\bar{\gamma}_{0}(x)=\bar{\gamma} J_{\Sigma}(x, t)$ from Equation (1.16), we then get

$$
-\int_{0}^{1} \int_{\Sigma} \bar{\gamma} J_{\Sigma} \partial_{t}^{2} \eta \cdot(v \circ \eta) d S d t
$$

which, using change of variables with the area form and substituting for the acceleration, is equal to

$$
-\int_{0}^{1} \int_{\Sigma(t)} \gamma \partial_{t}^{2} \eta \circ \eta(t)^{-1} \cdot v d S d t=-\int_{0}^{1} \int_{\Sigma(t)} \gamma\left(\partial_{t} u+u \cdot \nabla u\right) \cdot v d S d t .
$$

Looking at the third term, we have

$$
\begin{aligned}
\left.\partial_{s}\right|_{s=0} E_{3}(\zeta) & =\left.\partial_{s}\right|_{s=0} \int_{0}^{1} \int_{\Sigma} \xi\left(\bar{\gamma}_{0} J_{\Sigma}^{-1}\right) J_{\Sigma} d S d t=\int_{0}^{1} \int_{\Sigma} \xi(\bar{\gamma}) \partial_{s} J_{\Sigma}-\xi^{\prime}(\bar{\gamma}) \bar{\gamma}_{0} J_{\Sigma}^{-1} \partial_{s} J_{\Sigma} d S d t \\
& =\int_{0}^{1} \int_{\Sigma}\left[\xi(\bar{\gamma})-\xi^{\prime}(\bar{\gamma}) \bar{\gamma}\right] \partial_{s} J_{\Sigma} d S d t \\
& =\int_{0}^{1} \int_{\Sigma}\left[\xi(\bar{\gamma})-\xi^{\prime}(\bar{\gamma}) \bar{\gamma}\right]\left(\operatorname{div}_{\Sigma(t)}\left(\left(D \eta \partial_{s} \zeta\right) \circ \eta^{-1}\right) \circ \eta\right) J_{\Sigma} d S d t
\end{aligned}
$$

where change of variables by $\eta$ yields

$$
=\int_{0}^{1} \int_{\Sigma(t)}\left[\xi(\gamma)-\xi^{\prime}(\gamma) \gamma\right] \operatorname{div}_{\Sigma(t)}\left(\left(D \eta \partial_{s} \zeta\right) \circ \eta^{-1}\right) d S d t
$$

Recalling that we have $\sigma:=\xi(\gamma)-\xi^{\prime}(\gamma) \gamma$, we have

$$
\left.\partial_{s}\right|_{s=0} E_{3}(\eta)=-\int_{0}^{1} \int_{\Sigma(t)}\left(\nabla_{\Sigma(t)} \sigma+H \nu \sigma\right) \cdot\left(D \eta \partial_{s} \zeta\right) \circ \eta^{-1} d S d t
$$


Using Lemma 2.5 again, we know that $\left(D \eta \partial_{s} \zeta\right) \circ \eta^{-1}$ can be chosen to be any divergence free vector field $v$ and hence we have

$$
\left.\partial_{s}\right|_{s=0} E_{3}(\eta)=-\int_{0}^{1} \int_{\Sigma(t)}\left(\nabla_{\Sigma(t)} \sigma+H \nu \sigma\right) \cdot v d S d t
$$

Putting all three energies together, we thus find that

$$
\begin{aligned}
0 & =\left.\partial_{s}\right|_{s=0} E(\zeta) \\
& =\int_{0}^{1}\left(\int_{\Sigma(t)} \tilde{p} \nu \cdot v d S+\int_{\Sigma(t)}-\gamma\left(\partial_{t} u+u \cdot \nabla u\right) \cdot v d S+\int_{\Sigma(t)}\left(\nabla_{\Sigma(t)} \sigma+H \nu \sigma\right) \cdot v d S\right) d t
\end{aligned}
$$

We thus have for any divergence free $v$ that

$$
\int_{0}^{1} \int_{\Sigma(t)}\left(-\gamma\left(\partial_{t} u+u \cdot \nabla u\right)+\tilde{p} \nu+\nabla_{\Sigma(t)} \sigma+H \nu \sigma\right) \cdot v d S d t=0
$$

Now by Lemma 2.11, we know that there must exist $c_{0} \in \mathbb{R}$ that

$$
-\gamma\left(\partial_{t} u+u \cdot \nabla u\right)+\tilde{p} \nu+\nabla_{\Sigma(t)} \sigma+H \nu \sigma=c_{0} \nu
$$

Defining $p:=\tilde{p}-c_{0}$, we find that

$$
\gamma\left(\partial_{t} u+u \cdot \nabla u\right)-p \nu=\nabla_{\Sigma(t)} \sigma+H \nu \sigma
$$

By our assumption of conservation of mass, Equation (1.17), we have that, by changing variables twice,

$$
\begin{aligned}
0 & =\partial_{t} \int_{\eta(U, t)} \gamma d S=\partial_{t} \int_{U} \gamma(\eta(x, t), t) J_{\Sigma} d S=\int_{U}\left(\partial_{t} \gamma \circ \eta+\nabla \gamma \circ \eta \cdot \partial_{t} \eta\right) J_{\Sigma}+\gamma \circ \eta \partial_{t} J_{\Sigma} d S \\
& =\int_{U}\left(\partial_{t} \gamma \circ \eta+\nabla \gamma \circ \eta \cdot \partial_{t} \eta+\gamma \circ \eta\left(\operatorname{div}_{\Sigma(t)} u\right) \circ \eta\right) J_{\Sigma} d S \\
& =\int_{\eta(U, t)} \partial_{t} \gamma+\nabla \gamma \cdot v+\gamma \operatorname{div}_{\Sigma(t)} u d S .
\end{aligned}
$$

for any $U \subseteq \Sigma$. We conclude

$$
\partial_{t} \gamma+\nabla \gamma \cdot u+\gamma \operatorname{div}_{\Sigma(t)} u=0
$$

Putting this all together, we find

$$
\begin{cases}\rho\left(\partial_{t} u+u \cdot \nabla u\right)+\nabla p=-\nabla \varphi & \text { on } \Omega(t) \\ \operatorname{div} u=0 & \text { on } \Omega(t) \\ \gamma\left(\partial_{t} u+u \cdot \nabla u\right)-p \nu=\nabla_{\Sigma(t)} \sigma+H \nu \sigma & \text { on } \Sigma(t) \\ \partial_{t} \gamma+\nabla \gamma \cdot u+\gamma \operatorname{div}_{\Sigma(t)} u=0 & \text { on } \Sigma(t)\end{cases}
$$

as desired.

Finally, we prove Theorem 1.8.

Proof of Theorem 1.8. Similar to Theorem 1.7 we write $E$ as $E_{1}+E_{2}-E_{3}$ where

$$
\begin{aligned}
E_{1}(\eta) & :=\int_{0}^{1} \int_{\Omega} \frac{\bar{\rho}}{2}\left|\partial_{t} \eta\right|^{2}-\varphi(\eta) d x d t \\
E_{2}(\eta, \beta) & :=\int_{0}^{1} \int_{\Sigma} \frac{\bar{\gamma}_{0}}{2}\left|\partial_{t}(\eta \circ \beta)\right|^{2} d S d t \\
E_{3}(\eta, \beta) & :=\int_{0}^{1} \int_{\Sigma(t)} \xi(\gamma) d S d t
\end{aligned}
$$

Let $\zeta \in C^{\infty}((-\varepsilon, \varepsilon) ; \operatorname{FDiff}(\Omega)), B \in C^{\infty}((-\varepsilon, \varepsilon) ; Y)$ be arbitrary with $\zeta(0)=\eta$ and $B(0)=\beta$ (in other words, let $B$ be a perturbation of $\beta$ just as how $\zeta$ was a perturbation of $\eta$ ). 
We first only consider perturbations of $\eta$, which naturally yield calculations similar to Theorem 1.7. Reusing the calculation for $E_{1}$ from that result, we then find that the variation of the second term is

$$
\left.\partial_{s}\right|_{s=0} E_{2}(\zeta, \beta)=-\int_{0}^{1} \int_{\Sigma} \bar{\gamma}_{0} \partial_{t}^{2}(\eta \circ \beta) \cdot \partial_{s}(\eta \circ \beta) d S d t=-\int_{0}^{1} \int_{\Sigma} \bar{\gamma} J_{\Sigma} \partial_{t}^{2}(\eta \circ \beta) \cdot v \circ \eta \circ \beta d S d t
$$

By change of variables with $\eta \circ \beta$ and writing $\partial_{t}^{2}(\eta \circ \beta) \circ(\eta \circ \beta)^{-1}=\partial_{t} u_{s}+u_{s} \cdot \nabla u_{s}$, the above is equal to

$$
-\int_{0}^{1} \int_{\Sigma(t)} \gamma\left(\partial_{t} u_{s}+u_{s} \cdot \nabla u_{s}\right) \cdot v d S d t
$$

Looking at the third term, by a similar argument to Theorem 1.7, we have

$$
\begin{aligned}
\left.\partial_{s}\right|_{s=0} E_{3}(\zeta, \beta) & =\int_{0}^{1} \int_{\Sigma}\left[\xi(\bar{\gamma})-\xi^{\prime}(\bar{\gamma}) \bar{\gamma}\right] \partial_{s} J_{\Sigma} d S d t \\
& =\int_{0}^{1} \int_{\Sigma}\left[\xi(\bar{\gamma})-\xi^{\prime}(\bar{\gamma}) \bar{\gamma}\right] J_{\Sigma}\left(\operatorname{div}_{\Sigma(t)} v\right) \circ(\eta \circ \beta) d S d t
\end{aligned}
$$

where change of variable by $\eta \circ \beta$ and the surface divergence theorem (Theorem 2.9) yields

$$
\int_{0}^{1} \int_{\Sigma(t)} \sigma \operatorname{div}_{\Sigma(t)} v d S d t=-\int_{0}^{1} \int_{\Sigma(t)}\left(\nabla_{\Sigma(t)} \sigma+H \nu \sigma\right) \cdot v d S d t
$$

Putting this all together we have

$$
0=\left.\partial_{s}\right|_{s=0} E(\zeta, \beta)=\int_{0}^{1} \int_{\Sigma(t)}\left(\tilde{p} \nu-\gamma\left(\partial_{t} u_{s}+u_{s} \cdot \nabla u_{s}\right)+\nabla_{\Sigma(t)} \sigma+H \nu \sigma\right) \cdot v d S d t
$$

Thus by Lemma 2.11, for some $c_{0} \in \mathbb{R}$ we have

$$
\tilde{p} \nu-\gamma\left(\partial_{t} u_{s}+u_{s} \cdot \nabla u_{s}\right)+\nabla_{\Sigma(t)} \sigma+H \nu \sigma=c_{0} \nu
$$

Defining $p=\tilde{p}-c_{0}$, we find that

$$
\gamma\left(\partial_{t} u_{s}+u_{s} \cdot \nabla u_{s}\right)-p \nu=\nabla_{\Sigma(t)} \sigma+H \nu \sigma
$$

We now restrict attention to perturbations of $\beta$, finding that

$$
\begin{aligned}
\left.\partial_{s}\right|_{s=0} E_{2}(\eta, B) & =\int_{0}^{1} \int_{\Sigma} \bar{\gamma}_{0} \partial_{t}(\eta \circ \beta) \cdot \partial_{t}\left(D \eta \circ \beta \partial_{s} B\right) d S d t \\
& =-\int_{0}^{1} \int_{\Sigma} \bar{\gamma} J_{\Sigma} \partial_{t}^{2}(\eta \circ \beta) \cdot\left(D \eta \circ \beta \partial_{s} B\right) d S d t
\end{aligned}
$$

through integration by parts in time. Note that the boundary terms vanish because the perturbation is fixed at the boundary points. Now we change variables with $\eta \circ \beta$ and write $\partial_{t}^{2}(\eta \circ \beta) \circ(\eta \circ \beta)^{-1}=\partial_{t} u_{s}+u_{s} \cdot \nabla u_{s}$ so that the expression becomes

$$
\begin{aligned}
& =-\int_{0}^{1} \int_{\Sigma(t)} \gamma \partial_{t}^{2}(\eta \circ \beta) \circ(\eta \circ \beta)^{-1} \cdot\left(D \eta \circ \beta \partial_{s} B\right) \circ(\eta \circ \beta)^{-1} d S d t \\
& =-\int_{0}^{1} \int_{\Sigma(t)} \gamma\left(\partial_{t} u_{s}+u_{s} \cdot \nabla v_{s}\right) \cdot\left(D \eta \circ \beta \partial_{s} B\right) \circ(\eta \circ \beta)^{-1} d S d t
\end{aligned}
$$

where $u_{s}$ is the Eulerian velocity for surfactants.

Looking at the third term, by a similar argument in Theorem 1.7, we have

$$
\begin{aligned}
\left.\partial_{s}\right|_{s=0} E_{3}(\eta, B) & =\left.\partial_{s}\right|_{s=0} \int_{0}^{1} \int_{\Sigma} \xi\left(\bar{\gamma}_{0} J_{\Sigma}^{-1}\right) J_{\Sigma} d S d t=\int_{0}^{1} \int_{\Sigma} \xi(\bar{\gamma}) \partial_{s} J_{\Sigma}-\xi^{\prime}(\bar{\gamma}) \bar{\gamma}_{0} J_{\Sigma}^{-1} \partial_{s} J_{\Sigma} d S d t \\
& =\int_{0}^{1} \int_{\Sigma}\left[\xi(\bar{\gamma})-\xi^{\prime}(\bar{\gamma}) \bar{\gamma}\right] \partial_{s} J_{\Sigma} d S d t \\
& =\int_{0}^{1} \int_{\Sigma}\left[\xi(\bar{\gamma})-\xi^{\prime}(\bar{\gamma}) \bar{\gamma}\right]\left(\operatorname{div}_{\Sigma(t)}\left(\left(D(\eta \circ \beta) \partial_{s} B\right) \circ(\eta \circ \beta)^{-1}\right)\right) \circ(\eta \circ \beta) J_{\Sigma} d S d t
\end{aligned}
$$


where change of variable by $\eta \circ \beta$ yields

$$
=\int_{0}^{1} \int_{\Sigma(t)}\left[\xi(\gamma)-\xi^{\prime}(\gamma) \gamma\right] \operatorname{div}_{\Sigma(t)}\left(\left(D(\eta \circ \beta) \partial_{s} B\right) \circ(\eta \circ \beta)^{-1}\right) d S d t
$$

Recalling that we define $\sigma:=\xi(\gamma)-\xi^{\prime}(\gamma) \gamma$, we have

$$
\left.\partial_{s}\right|_{s=0} E_{3}(\eta, B)=-\int_{0}^{1} \int_{\Sigma(t)}\left(\nabla_{\Sigma(t)} \sigma+H \nu \sigma\right) \cdot\left(D(\eta \circ \beta) \partial_{s} B\right) \circ(\eta \circ \beta)^{-1} d S d t .
$$

Putting these together,

$$
0=\int_{0}^{1} \int_{\Sigma(t)}\left(-\gamma\left(\partial_{t} u_{s}+u_{s} \cdot \nabla u_{s}\right)+\nabla_{\Sigma(t)} \sigma+H \nu \sigma\right) \cdot\left(D(\eta \circ \beta) \partial_{s} B\right) \circ(\eta \circ \beta)^{-1} d S d t .
$$

Note $B$ is a one-parameter family of diffeomorphisms of $\Sigma$. By choosing arbitrary perturbation, $\partial_{s} B$ can represent any vector field on $\Sigma$, thus $D(\eta \circ \beta) \partial_{s} B$ can represent any vector field on $\Sigma(t)$.

Hence Equation (3.25) implies

$$
-\gamma\left(\partial_{t} u_{s}+u_{s} \cdot \nabla u_{s}\right)+\nabla_{\Sigma(t)} \sigma+H \nu \sigma
$$

only has normal components, i.e.

$$
-\gamma\left(\partial_{t} u_{s}+u_{s} \cdot \nabla u_{s}\right)+\nabla_{\Sigma(t)} \sigma+H \nu \sigma=\lambda(x, t) \nu
$$

for some scalar function $\lambda: \Sigma(t) \times[0,1] \rightarrow \mathbb{R}$.

But this coupled with Equation (3.23) immediately implies that $\lambda=p$.

Finally as in Theorem 1.7, we have preservation of mass

$$
\partial_{t} \gamma+\nabla \gamma \cdot u+\gamma \operatorname{div}_{\Sigma(t)} u=0
$$

Putting this all together, we find

$$
\begin{cases}\rho\left(\partial_{t} u+u \cdot \nabla u\right)+\nabla p=-\nabla \varphi & \text { on } \Omega(t) \\ \operatorname{div} u=0 & \text { on } \Omega(t) \\ \gamma\left(\partial_{t} u_{s}+u_{s} \cdot \nabla u_{s}\right)-p \nu=\nabla_{\Sigma(t)} \sigma+H \nu \sigma & \text { on } \Sigma(t) \\ \partial_{t} \gamma+\nabla \gamma \cdot u+\gamma \operatorname{div}_{\Sigma(t)} u=0 & \text { on } \Sigma(t)\end{cases}
$$

\section{ACKNOWLEDGMENTS}

We would like to thank advisor Ian Tice for his guidance and mentorship throughout this project. We deeply appreciate the generosity with which he has given us his time and energy. We would like to thank the anonymous referees for their careful reading of the first draft and for their many helpful suggestions. This project was supported by funding from the NSF CAREER grant (\#1653161).

\section{REFERENCES}

[AK98] V. I. Arnold and B. A. Khesin. Topological Methods in Hydrodynamics. Appl. Math. Sci., v. 125. Springer, New York, First edition, 1998.

[Arn66] V. I. Arnold. On the differential geometry of infinite-dimensional lie groups and its application to the hydrodynamics of perfect fluids. In Givental A. et al, editor, Vladimir I. Arnold - Collected Works, vol 2., pages 33-69. Springer, 1966.

[BF13] F. Boyer and P. Fabrie. Mathematical tools for the study of the incompressible Navier-Stokes equations. Appl. Math. Sci., v. 183. Springer, New York, 2013.

[EBW91] D. A. Edwards, H. Brenner, and D. T. Wasan. Interfacial Transport Processes and Rheology. Reed Publishing Inc (USA), Stonheam, MA, 1991.

[Eva10] L. C. Evans. Partial differential equations. Graduate studies in mathematics, v. 19. J. Amer. Math. Soc., Providence, R.I, Second edition, 2010.

[Hil99] B. Hills. An alternative view of the role(s) of surfactant and the alveolar model. J. Appl. Physiol., 87:1567-1583, 1999. 
[HLT08] H. Huang, M-C. Lai, and H-C. Tseng. A parametric derivation of the surfactant transport equation along a deforming fluid interface. Front. Appl. Comput. Math., January 2008.

[KMM20] Boris Khesin, Gerard Misiolek, and Klas Modin. Geometric hydrodynamics and infinitedimensional newton's equations, 2020.

[LY02] F. Lin and X. Yang. Geometric Measure Theory - An Introduction. International Press, Cambridge, MA, 2002.

[Omo74] H. Omori. Infinite Dimensional Lie Transformation Groups. Lecture Notes in Mathematics, 427. Springer Berlin Heidelberg, Berlin, Heidelberg, 1st ed. 1974. edition, 1974.

[Sar96] T. Sarpkaya. Vorticity, free surface, and surfactants. Annual Review of Fluid Mechanics, 28:83-128, 1996.

[Sch87] R. Schmid. Infinite dimensional hamiltonian systems with symmetries. 1987.

[Sch95] G Schwarz. Hodge Decomposition-A Method for Solving Boundary Value Problems. Lecture Notes in Mathematics. Springer, Mannheim, First edition, 1995.

[Sto90] H. A Stone. A simple derivation of the time-dependent convective-diffusion equation for surfactant transport along a deforming interface. Physics of fluids. A, Fluid dynamics, 2(1):111-112, 1990.

[SZ07] J. Shatah and C. Zeng. Geometry and a priori estimates for free boundary problems of the euler equation. Comm. Pure Appl. Math., Vol. LXI:698-744, 2007.

[TW17] I. Tice and L. Wu. Dynamics and stability of surface waves with bulk-soluble surfactants. Acta Appl. Math, 161:35-70, February 2017. 